\title{
The evolution of multiple-insecticide resistance in UK populations of tomato leafminer, Tuta absoluta
}

\author{
Charles Grant, ${ }^{a}$ Rob Jacobson, ${ }^{b}$ Aris Ilias, ${ }^{c}$ Madeleine Berger, ${ }^{d}$ \\ Emmanouil Vasakis, ${ }^{e}$ Pablo Bielza, ${ }_{\oplus} \oplus C$ Christoph T Zimmer, ${ }^{a \dagger}$ \\ Martin S Williamson, ${ }^{d}$ Richard H ffrench-Constant, ${ }^{a}$ John Vontas, ${ }^{c, g}$ \\ Emmanouil Roditakis ${ }^{\mathrm{e}}$ and Chris Bass $^{\mathrm{a}^{*}}{ }^{\circ}$
}

\section{Abstract}

BACKGROUND: The tomato leafminer, Tuta absoluta, is an economically important pest of tomatoes in Europe, Africa, Asia and South America. In the UK this species is controlled using an integrated pest management (IPM) programme which incorporates the insecticides spinosad and chlorantraniliprole. In response to UK grower concerns of loss of efficacy of these compounds at certain sites, insecticide bioassays were performed on five populations collected from four commercial glasshouses and potential mechanisms of resistance investigated.

RESULTS: We observed high levels of resistance to spinosad in four of the strains, and in two of these tolerance to chlorantraniliprole. Selection of one of these strains with chlorantraniliprole rapidly resulted in a line exhibiting potent resistance to this compound. Sequencing of messenger RNA encoding the nicotinic acetylcholine receptor (nAChR) $\alpha 6$ subunit, target of spinosad, revealed Ta 6 transcripts in the spinosad-resistant strains that lack exon 4 and encode a highly truncated protein, or contain a triplet deletion in the predicted first transmembrane domain resulting in the loss of a highly conserved amino acid. Sequencing of the ryanodine receptor gene, encoding the target of diamide insecticides, of the chlorantraniliprole-selected line revealed an amino acid substitution (G4903V) that has been previously linked to diamide resistance in populations of $T$. absoluta in the Mediterranean and South America.

CONCLUSION: Taken together our results reveal emerging resistance in UK populations of $T$. absoluta to two of the most important insecticides used as part of IPM, with significant implications for the control of this species in the UK.

(c) 2019 Society of Chemical Industry

Supporting information may be found in the online version of this article.

Keywords: spinosad; chlorantraniliprole; resistance; tomato leafminer

\section{INTRODUCTION}

The tomato leafminer, Tuta absoluta, is an economically important pest of tomatoes which spread from South America to Spain in 2006, subsequently invading a range of other European countries, Africa and the Middle East. ${ }^{1}$ T. absoluta arrived in the UK in $2009^{2}$ and caused profound damage to commercial tomato crops at sites across the country before an integrated pest management (IPM) strategy was developed that provided effective control. ${ }^{3}$ This IPM strategy integrated biological control using the predatory bug Macrolophus pygmaeus, with some physical control measures and three insecticides: the spinosyn spinosad, the diamide chlorantraniliprole and the oxadiazine indoxacarb. These three insecticides were selected in part due to their different modes of action in order to prevent or delay the emergence of resistance. Recently, however, some UK growers have reported a loss of efficacy of both spinosad and chlorantraniliprole against T. absoluta (R. Jacobson personal communication). While, to date, resistance in UK populations of T. absoluta to these compounds has not
Correspondence to: C Bass, College of Life and Environmental Sciences, Biosciences, University of Exeter, Penryn Campus, Penryn, Cornwall, UK. E-mail:c.bass@exeter.ac.uk

$\dagger$ Present address: Syngenta Crop Protection, Werk Stein, Schaffhauserstrasse, Stein $\mathrm{CH} 4332$, Switzerland.

a College of Life and Environmental Sciences, Biosciences, University of Exeter, Penryn Campus, Penryn, UK

b RJCLtd, Bramham, UK

c Institute of Molecular Biology \& Biotechnology, Foundation for Research \& Technology Hellas, Crete, Greece

d Department of Biointeractions and Crop Protection, Rothamsted Research, Harpenden, UK

e Hellenic Agricultural Organisation - 'Demeter', Institute of Olive Tree, Subtropical Crops and Viticulture, Heraklion, Greece

f Departamento de Producción Vegetal, Universidad Politécnica de Cartagena, Cartagena, Spain

g Department of Crop Science, Agricultural University of Athens, Athens, Greece 
been confirmed, both resistance and the underlying mechanisms involved have been characterised in populations of $T$. absoluta in other countries. In the case of spinosad, resistance has been described in field populations from Brazil with subsequent selection of one of these strains resulting in a line with extremely high levels of resistance to this compound. ${ }^{4}$ Spinosad acts on the insect nervous system by targeting the $\alpha 6$ subunit of the nicotinic acetylcholine receptor (nAChR), a neurotransmitter-gated ion channel that plays a critical role in nerve signalling at the postsynaptic membrane. Sequencing of the gene encoding this subunit in the spinosad selected strain identified a point mutation in exon 9 of Ta 6 that results in a G275E amino acid substitution. ${ }^{5}$ The causal role of this mutation in resistance was subsequently confirmed by CRISPR/Cas9 gene editing. ${ }^{6}$ In a second study, characterisation of the Ta $\alpha 6$ gene in a resistant strain selected from a field population collected in Portugal revealed an alternative mechanism of resistance conferred by skipping of exon 3 in transcripts of Ta $6 .{ }^{7}$

Resistance to chlorantraniliprole has been recently described in populations of T. absoluta from Italy, Greece, Spain and Brazil..$^{8-10}$ Sequencing of the gene encoding the ryanodine receptor (RyR), a ligand-gated calcium-channel located in the sarco- and endoplasmic reticulum of neuromuscular tissues, identified several target-site mutations in the resistant strains. ${ }^{8}$ Two of these, G4903E and I4746M, had been previously identified in diamide resistant diamondback moth, ${ }^{11,12}$ while two others, G4903V and I4746T, were novel to T. absoluta. Radioligand binding studies and CRISPR/Cas9 gene editing provided unequivocal functional evidence that these mutations alter the affinity of the RyR to diamides and confer resistance. $8,13,14$

The resistance mechanisms described above have not yet been described in UK populations of T. absoluta. However, the rapid invasion of this species into the UK from Europe suggests that European populations carrying resistance alleles may be readily imported into the UK, especially as T. absoluta is no longer a notifiable (quarantine) pest in the UK. Alternatively, the use of spinosad and chlorantraniliprole in the UK for the control of $T$. absoluta might lead to the de novo emergence of resistance. In the current study we explored these possibilities by carrying out phenotypic and molecular characterisation of resistance in several UK populations of T. absoluta.

\section{MATERIALS AND METHODS}

\subsection{Insect strains}

After reports of control failure using spinosad, four strains of $T$. absoluta were collected in 2015 from commercial glasshouses at four UK sites: IOW from Wight salads, Isle of Wight, LAN from Flavourfresh Salads Ltd, Lancashire, NY from Jan Bezemer and Sons, North Yorkshire and EVH from R and L Holt, near Evesham, Worcestershire. In 2016 a second strain, EVH16, was collected from $\mathrm{R}$ and $\mathrm{L}$ Holt after reports of control failure using chlorantraniliprole. An additional Spanish strain that is susceptible to spinosad and chlorantraniliprole was acquired from Rothamsted Research, Hertfordshire. Insects were housed in controlled environment rooms at $25^{\circ} \mathrm{C}, 60 \% \mathrm{RH}, 16: 8$ light-dark cycle and fed ad libitum on tomato plants (var. Money Maker).

\subsection{Leaf-dip assays}

Leaf-dip bioassays were completed according to test method 22 guidelines produced by the Insecticide Resistance Action Committee. ${ }^{15}$ Probit analysis and the calculation of $\mathrm{LC}_{50}$ values were performed with GenStat 15th Edition (VSN International, Hemel Hempstead, UK).

\subsection{Chlorantraniliprole selection}

Infested tomato leaves containing in excess of 500 larvae of the EVH16 strain were taken from cages and placed on a course net suspended over tomato branches that had been treated with chlorantraniliprole. This was repeated over the course of 12 months at concentrations of 1,5, 10 and $50 \mathrm{ppm}$. After each round of selection the line was left unselected for two full generations to allow the population to recover. The resulting selected line was named EVH16sel.

\subsection{RNA extraction and cDNA synthesis}

T. absoluta L2-L4 larvae were snap frozen in liquid nitrogen and stored at $-80^{\circ} \mathrm{C}$ prior to RNA extraction. RNA extractions were carried out using the ISOLATE II RNA Mini Kit (Bioline, London, UK) on individual larvae or pools of ten larvae. CDNA was then synthesized from $1 \mu \mathrm{g}$ of total RNA using the Maxima H Minus First Strand CDNA Synthesis kit (Thermo Fisher Scientific, Waltham, US) according to the manufacturer's protocol.

\subsection{Amplification and sequencing of the RyR and nAChR $\alpha 6$ subunit}

A 1353 bp region of the T. absoluta RyR gene (encompassing the sites of previously reported diamide resistance-conferring mutations) was amplified by PCR using DreamTaq Green PCR master mix (Thermo Fisher Scientific, Waltham, US) and the primers detailed in Table S1. Primers were designed based on the previously published T. absoluta RyR mRNA sequence. ${ }^{8}$ Ten nanograms ( $\left.1 \mu \mathrm{L}\right)$ of cDNA was used as template. Temperature cycling conditions comprised 3 min at $95^{\circ} \mathrm{C}$ followed by 35 cycles of $95^{\circ} \mathrm{C}$ for $30 \mathrm{~s}, 54.5^{\circ} \mathrm{C}$ for $30 \mathrm{~s}$ and $72{ }^{\circ} \mathrm{C}$ for $60 \mathrm{~s}$, and a final extension step of $72{ }^{\circ} \mathrm{C}$ for $6 \mathrm{~min}$. Amplification of the $\mathrm{nAChR} \alpha 6$ subunit gene was performed using the primers detailed in Table S1 designed on the previously published Ta $\alpha 6$ mRNA sequence. ${ }^{7}$ A nested PCR protocol was used with $10 \mathrm{ng}(1 \mu \mathrm{L})$ of CDNA used in the primary PCR, and $1 \mu \mathrm{L}$ of a 1:10 dilution of the product used in a secondary reaction. Temperature cycling conditions were as above. PCR products were purified using the GeneJET PCR purification kit (Thermo Fisher Scientific) and purified DNA quantified by spectrophotometry (NanoDrop, Thermo Fisher Scientific, Waltham, US) prior to Sanger sequencing using the primers detailed in Table S1.

\subsection{Association of F238del with spinosad resistance}

To examine the association of the F238del mutation and resistance to spinosad we utilised the LAN strain as it is heterogeneous for this mutation (see Results). Twenty replicates of ten larvae of this strain were placed on tomato leaves treated with $150 \mathrm{ppm}$ of spinosad. As a control five replicates of ten larvae were placed on a tomato leaf treated only with the insecticide dilutant. Mortality was scored after $72 \mathrm{~h}$ and all larvae were snap-frozen in liquid nitrogen and stored at $-80^{\circ} \mathrm{C}$. DNA was subsequently extracted from over 200 individual larvae using DNAzol following the manufacturer's protocol. A TaqMan SNP genotyping assay was designed using the Custom TaqMan Assay Design Tool (Applied Biosystems Foster (ity, US) and used to score the genotypes of dead and alive individuals generated above for the F238del mutation. Primers and minor groove binder (MGB) probes are detailed in Table S1. Each probe was labeled with a $5^{\prime}$ reporter dye specific to either 
the wild-type or mutant allele. The VIC reporter dye was used to detect the wild-type allele and the 6FAM reporter dye to detect the mutant allele. Each probe also carried a 3' non-fluorescent quencher and a minor groove binder at the $3^{\prime}$ end that provides more accurate allelic discrimination by increasing the melting temperature (TM) between matched and mis-matched probes. PCR reactions contained $2 \mu \mathrm{L}$ genomic DNA, extracted from individual insects using DNAzol reagent, $7.5 \mu \mathrm{L}$ of SensiFAST SYBR No-ROX kit (Bioline), $800 \mathrm{~nm}$ of each primer and $200 \mathrm{~nm}$ of each probe. Samples were run on a CFX96 real-time PCR machine (Bio-Rad Hercules, US) using the temperature cycling conditions of $10 \mathrm{~min}$ at $95^{\circ} \mathrm{C}$ followed by 40 cycles of $95^{\circ} \mathrm{C}$ for $10 \mathrm{~s}$ and $60^{\circ} \mathrm{C}$ for $45 \mathrm{~s}$. The increase in fluorescence of VIC and FAM was monitored in real time. In all runs, at least one control for each genotype (mutant homozygous, heterozygous and wild-type homozygous) was included. Pearson chi-square test was performed to examine the association between the F238del mutation and survival to spinosad exposure. To analyse the individual interactions between phenotype and genotype post-hoc analyses were conducted with adjusted residuals converted to a chi-square score and compared to a Bonferroni adjusted $\alpha$. Chi-square and post-hoc analyses were completed in SPSS (ver. 22, IBM Corp. Armonk, US).

\section{RESULTS}

\subsection{Sensitivity of T. absoluta strains to spinosad and chlorantraniliprole}

The sensitivity of the five UK strains of T. absoluta $(\mathrm{EVH}, \mathrm{EVH} 16$, LAN, IOW and NY) to spinsoad and chlorantraniliprole was compared to a reference susceptible strain (TA1) from Spain by full dose-response bioassays. In the case of spinosad significant differences in the $L_{50}$ values obtained were observed between four of the UK strains (EVH, EVH16, LAN and NY) and TA1 with resistance ratios ranging from 29 to $>480$ (Table 1). The LAN strain exhibited moderate resistance to spinosad with an $\mathrm{LC}_{50}$ of $149 \mathrm{mg} \mathrm{L}^{-1}$ while the EVH, EVH16 and NY strains showed high levels of resistance to this compound with $\mathrm{LC}_{50}$ values of $860,>500$ and $>2500 \mathrm{mg} \mathrm{L}^{-1}$, respectively (Table 1 ). Just two of the UK strains, NY and EVH16, exhibited tolerance to chlorantraniliprole. The $\mathrm{LC}_{50}$ of the two strains was 8-28-fold higher than that of the susceptible TA1 strain. Selection of the NY strain with chlorantraniliprole failed to rapidly result in a strain with higher levels of resistance. However, progressive selection of the EVH strain with chlorantraniliprole over just 12 generations resulted in a strain with potent resistance to this compound $\left(\mathrm{LC}_{50}\right.$ of $\left.>5000 \mathrm{mg} \mathrm{L}^{-1}\right)$.

\subsection{Identification of altered nAChR $\alpha 6$ transcripts in spinosad resistant strains of replace with $T$. absoluta}

Amplification and sequencing of the $\mathrm{nAChR} \alpha 6$ mRNA revealed the presence of two altered Ta $\alpha 6$ transcripts in the four resistant strains that were not observed in the susceptible strains TA1 and IOW. The first of these, observed in all Ta $\alpha 6$ transcripts of the NY, EVH and EVH16 strains was a deletion of $109 \mathrm{bp}$ that precisely corresponds to the position and size of exon 4 . This deletion results in a frameshift leading to the introduction of a premature termination codon (PTC) in exon 5 (Fig. 1). Amplification and sequencing of the Ta 6 gene from genomic DNA of the NY and EVH strains revealed that exon 4 was present and unaltered in both strains, confirming that the loss of this exon in mRNA results from exon skipping rather than a deletion of the exon in genomic DNA.

The second alteration, observed only in the LAN strain, was an in-frame triplet deletion resulting in the predicted loss of a phenylalanine amino acid at position 238 (T. absoluta numbering) (Fig. 1). The F238del mutation occurs in the first $\alpha$-helical transmembrane domain (TM1) of the Ta $\alpha 6$ subunit and comparison of insect nAChR $\alpha 6$ sequences from a range of insect species (Fig. 1) demonstrates that this residue is highly conserved across the Arthropoda. Unlike exon 4 skipping this alteration was observed in both the Ta $\alpha 6$ gene and mRNA transcript.

To examine the association of the F238del mutation with spinosad resistance the LAN strain was treated with a discriminating dose of spinosad and alive, dead and affected (larvae that were alive but unable to crawl their own body length away from mechanical pressure) larvae genotyped. Of the 200 individuals exposed to $150 \mathrm{mg} \mathrm{L}^{-1}$ of spinosad 108 died, 30 were affected, 55 survived the treatment and 7 escaped. To rapidly genotype these individuals, and to provide a diagnostic tool for future resistance monitoring studies, a TaqMan SNP genotyping assay was designed. This assay showed excellent discrimination of the resistant and susceptible alleles using two fluorescently labelled probes when tested with sequence verified DNA samples (Fig. S1).

Table 1. Sensitivity of UK strains of T. absoluta to the insecticides spinosad and chlorantranilprole. The resistance ratio (RR) of these strains was compared to a susceptible strain from Spain (TA1)

\begin{tabular}{|c|c|c|c|c|c|}
\hline Treatment & Strain & Location & $\mathrm{LC}_{50}\left(\mathrm{mg} \mathrm{L}^{-1}\right)$ & $95 \% \mathrm{Cl}$ & $\mathrm{RR}$ \\
\hline \multirow[t]{6}{*}{ Spinosad } & TA1 & Spain & 5.2 & $3.1-7.3$ & 1 \\
\hline & NY & North Yorkshire & $>2500$ & & $>481$ \\
\hline & EVH & Evesham & 860 & $484-2114$ & 165.38 \\
\hline & EVH16 & Evesham & $>500$ & & $>96$ \\
\hline & LAN & Lancashire & 149 & $31-5385$ & 28.65 \\
\hline & IOW & Isle of Wight & 8.6 & $5.3-13.9$ & 1.65 \\
\hline \multirow[t]{7}{*}{ Chlorantraniliprole } & TA1 & Spain & 1.2 & $0.095-5.00$ & 1 \\
\hline & NY & North Yorkshire & 33 & $11.2-97.9$ & 27.5 \\
\hline & EVH & Evesham & 0.12 & $0.005-1.00$ & 0.1 \\
\hline & EVH16 & Evesham & 9.53 & $5.025-18.09$ & 7.94 \\
\hline & EVH16sel & Evesham & $>5000$ & & $>4167$ \\
\hline & LAN & Lancashire & 4.75 & $1.7-12.8$ & 3.96 \\
\hline & IOW & Isle of Wight & 3.6 & $0.9-14$ & 3 \\
\hline
\end{tabular}




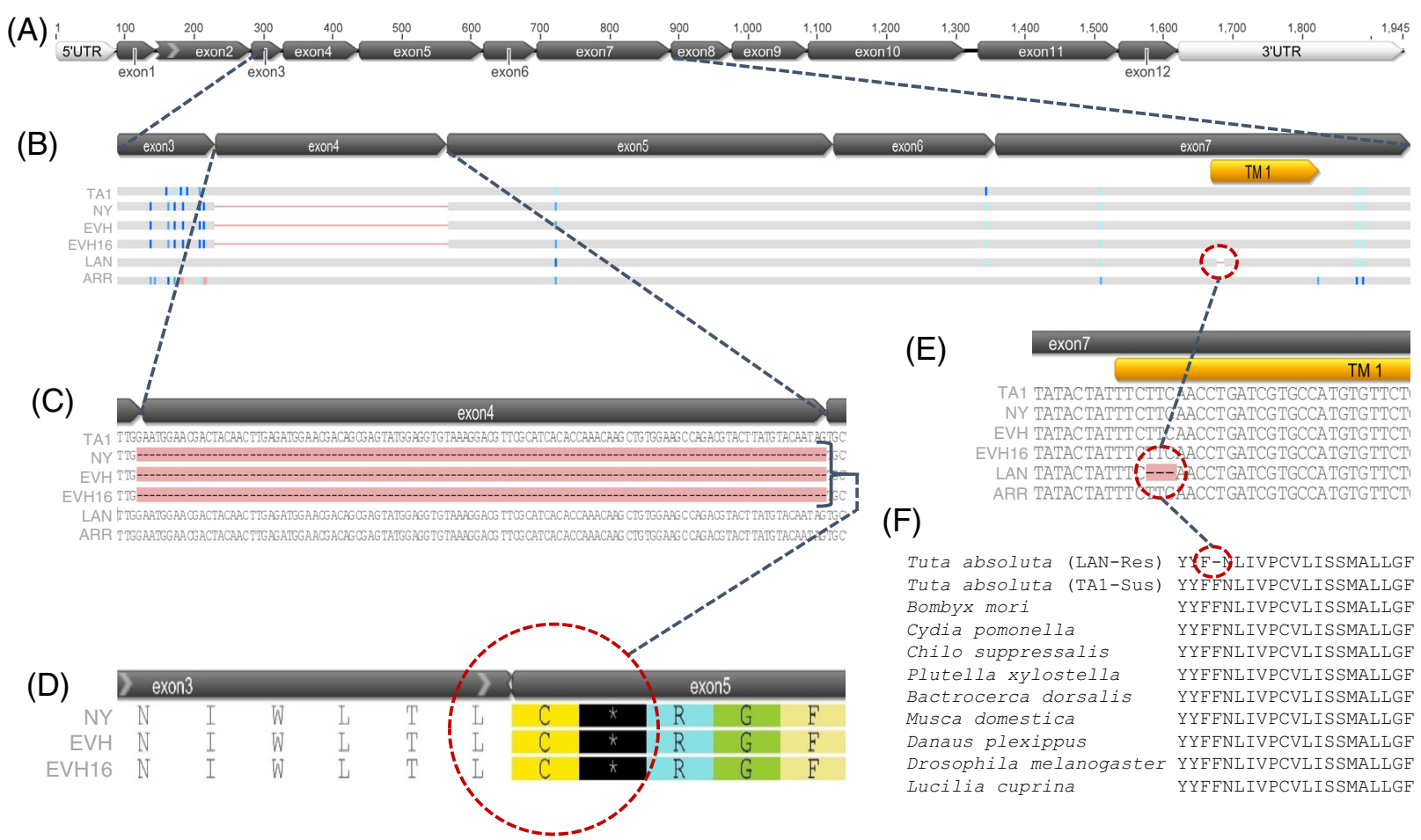

Figure 1. Schematic of mutations identified in Ta 6 transcripts of spinosad-resistant T. absoluta strains. (A) Schematic of the exon structure of Ta 6 (defined previously ${ }^{7}$ ). (B-D) Alignment of the sequences obtained from six T. absoluta strains illustrating the skipping of exon 4 observed in three of the spinosad-resistant strains that leads to the introduction of a premature termination codon in exon 5. (E, F) The LAN strain has a triplet deletion in the encoded first transmembrane domain of Ta $\alpha 6$ that leads to an in-frame deletion of an amino acid that is highly conserved in nAChR $\alpha 6$ subunit sequences across Arthropoda.

One hundred and seventy five individuals from the discriminating dose assay were successfully genotyped using this assay, with 31 individuals scored as homozygous for the wildtype allele, 56 as homozygous for the mutant allele and 88 heterozygous, indicating an overall mutation frequency of 0.57 in the LAN strain. A significant association was observed between the individual's genotype and its response to spinosad exposure $\left(\chi^{2}(4)=78.499\right.$, $P<0.001$ ). Post-hoc analysis (Table S2) revealed that none of the 'insecticide affected' group showed a statistically significant deviation from the proportion of genotypes expected by chance. However, individuals that died in the assay had a lower than expected proportion of the RR genotype $(P<0.001)$ and a greater than expected proportion of SS $(P<0.001)$ and RS genotypes $(P<0.001)$. In contrast, individuals surviving the assay had a higher than expected proportion of the RR genotype $(P<0.001)$ and a lower proportion of SS $(P<0.001)$ and RS $(P<0.001)$ genotypes (Fig. 2).

A 1353 bp region of the RyR encompassing the sites of mutations previously shown to confer resistance to diamides in T. absoluta ${ }^{8}$ and Plutella xylostella ${ }^{12}$ (G4903E/V and I4746M/T in T. absoluta corresponding to G4946E and I4790M in P.xylostella) was amplified from the chlorantraniliprole selected line (EVH16sel), the parent strain from which it was derived, (EVH16), NY and TA1 (Fig. 3). The sequence obtained from a pooled sample of the parental EVH16 strain encoded the wildtype amino acid at all the positions detailed above, however, a very small secondary peak was observed in the sequence chromatogram at position 4903, suggesting the G4903V substitution was present at very low frequency in this strain (Fig. 3). This was confirmed when the RyR sequence from the selected line was examined, which showed fixation of the mutation leading to this amino acid substitution.

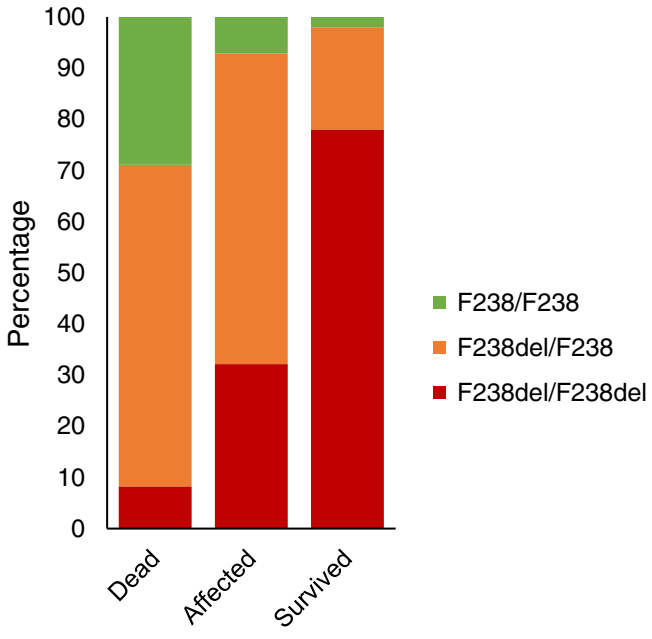

Response to spinosad

Figure 2. Genotype of T. absoluta larvae for the F238del mutation that died survived or were affected following exposure to a discriminating dose of spinosad. $n$ dead $=108, n$ affected $=30, n$ survived $=55$.

No mutations were observed in RyR sequences of the NY and TA1 strains.

\section{DISCUSSION}

Our data reveal high levels of spinosad resistance in strains of $T$. absoluta collected from nurseries in the north and south of the 


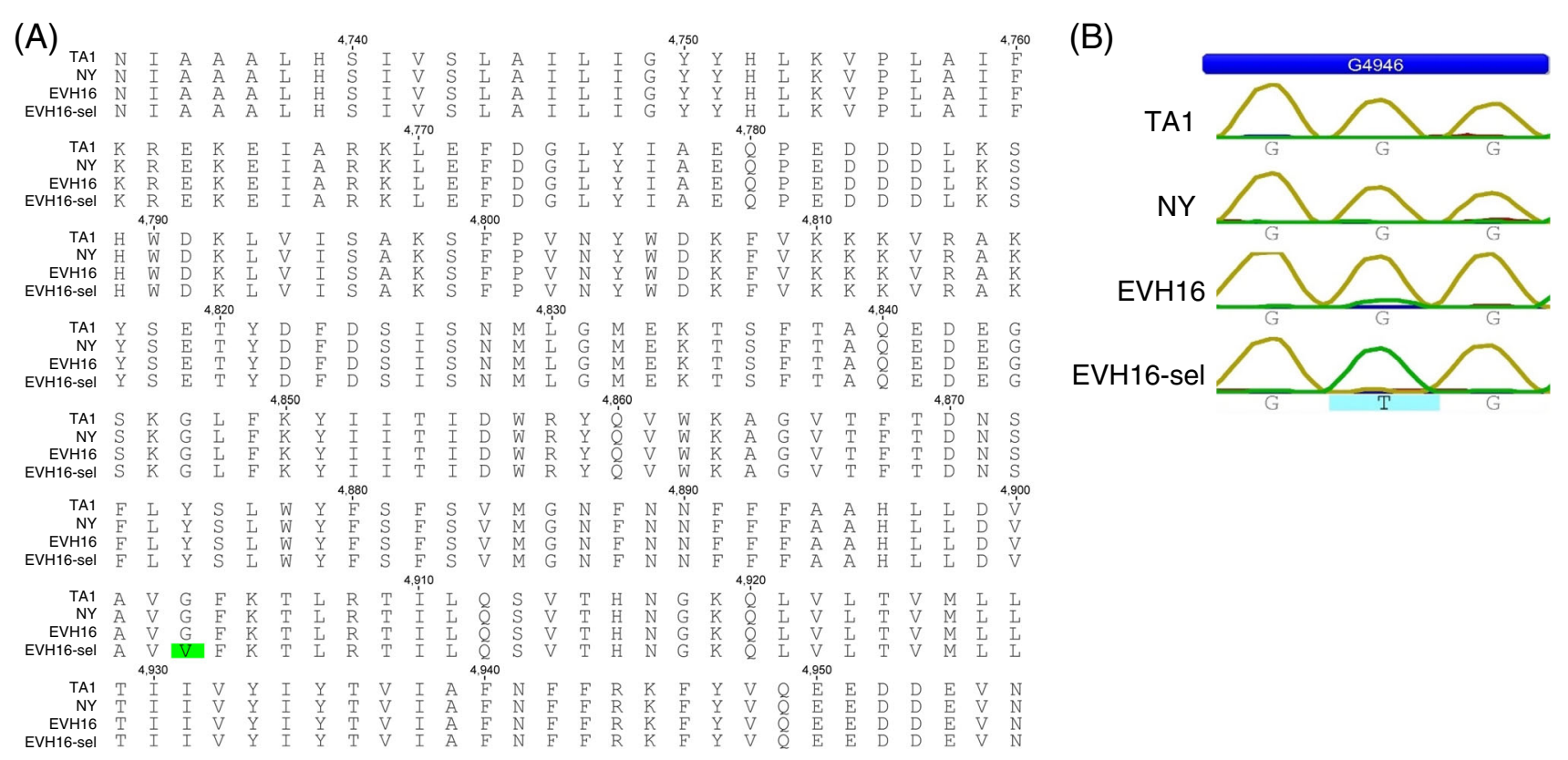

Figure 3. Identification of the G4903V amino acid substitution in a diamide-resistant strain of T. absoluta. (A) Alignment of RyR amino acid sequences obtained from three UK strains of T. absoluta and a strain from Spain. (B) Sequence chromatograms of the same strains at the G4903 codon illustrating a slight secondary ' $\mathrm{T}$ ' peak in the second nucleotide of the codon EVH16 strain that has become fixed following chlorantraniliprole selection (EVH16sel).

UK, indeed, only one of the UK strains, sampled from the Isle of Wight, exhibited full susceptibility to this compound. While our sample size is small this finding is consistent with reports by several British tomato growers of poor efficacy of spinosad against $T$. absoluta and suggests that resistance to this insecticide may be widespread in UK populations. In three of the strains, NY, EVH and EVH16, the levels of resistance observed were extremely high and would certainly be expected to compromise the use of spinosad at the recommended field rate $\left(87-100 \mathrm{mg} \mathrm{L}^{-1}\right)$. Lower levels of resistance were observed in the $L A N$ strain, however, the $L C_{50}$ value determined for this strain indicates that a significant percentage of individuals would be expected to survive exposure to the field rate application.

Sequence characterisation of transcripts encoding the $\mathrm{nAChR}$ a6 subunit revealed two novel mutations in the spinosad-resistant strains. The first of these, observed in the three most resistant strains, is skipping of exon 4. This alteration has profound consequences for the encoded protein, which would lack the $~ 36$ amino acids encoded by exon 4, and would also be significantly truncated as the loss of exon 4 leads to a frameshift and the introduction of a PTC in exon 5. Together this would result in a protein of just 160 amino acids (compared to 510 amino acids in the wildtype) that lacks all four transmembrane domains and would almost certainly be non-viable. Work on Drosophila melanogaster first demonstrated that null mutations of $\alpha 6$ lead to spinosad resistance with a strain with a variant of Da 6 lacking the TM3 and TM4 cytoplasmic loops, and the extracellular C-terminal tail domains exhibiting $>1000$-fold levels of resistance to spinosad. ${ }^{16}$ Significantly, this study also demonstrated that loss-of-function mutations in insect $\alpha 6$ transcripts do not result in catastrophic loss of fitness as insects without a functional copy of the $\alpha 6$ gene are still viable. ${ }^{16}$ Subsequent studies on several insect crop pests have described genetic alterations in $\mathrm{nAChR} \alpha 6$ sequences that result in truncated non-functional proteins leading to resistance. For example, several mutations resulting in mis-splicing and the introduction of PTCs in $\mathrm{nAChR} \alpha 6$ transcripts are associated with spinosad resistance in the diamondback moth, Plutella xylostella, ${ }^{17,18}$ the oriental fruit fly Bactrocera dorsalis, ${ }^{19}$ the western flower thrips, Frankliniella occidentalis $^{20}$ and the lesser grain borer, Rhyzopertha dominica. ${ }^{21}$ In a previous study of a spinosad resistant $T$. absoluta strain, derived from a population collected in Portugal, resistance was conferred by skipping of exon 3 rather than exon 4, however, in this case the reading frame of the altered Ta $\alpha 6$ transcripts was unaffected by the exclusion of exon 3.7 Finally, it is worth noting that while there is parity in the frequency of exon 4 skipping in the NY, EVH and $\mathrm{EVH} 16$ strains some variation was seen in their respective sensitivity to spinosad (Table 1), with the NY strain exhibiting the greatest resistance to this compound. This suggests that other additional mechanisms of resistance may be present in the NY strain, and it would be interesting to explore the potential role of metabolic mechanisms in the resistance of this strain to spinosad in future studies.

A more subtle alteration, F238del, was observed in transcripts of Ta 6 in the LAN strain and this was correlated with lower levels of resistance to spinosad. This triplet deletion results in the loss of a phenylalanine amino acid in exon 7 and occurs in TM1 of the nAChR Ta $\alpha 6$ subunit. This amino acid is highly conserved in $\mathrm{nAChR} \alpha 6$ subunit proteins across Arthropoda, suggesting that this residue is functionally constrained. The loss of the amino acid at this position is thus extremely unusual and consistent with it being a bona fide resistance mutation. We provide further evidence of a causal role of this mutation in resistance by demonstrating that it cosegregates with spinosad resistance in survival bioassays. The loss of one or more amino acids in the nAChR $\alpha 6$ subunit has been linked to spinosad resistance previously with a three amino acid deletion in the fourth transmembrane region of the receptor shown to confer resistance in P. xylostella. ${ }^{22}$ Furthermore, the F238del mutation exhibits parallels with a previously reported amino acid substitution (G275E, P. xylostella numbering) shown to confer spinosad resistance in both thrips and T. absoluta 
(the latter in strains from Brazil).5,6,23-25 Like F238del, this mutation also occurs in a $\alpha$-helical transmembrane domain (TM3) of the $\alpha 6$ subunit. ${ }^{24} \mathrm{X}$-ray crystallisation studies of the glutamate-gated chloride channel (GluCl), a pentameric receptor with close structural similarity to $\mathrm{nAChRs}$, bound to ivermectin, another macrocyclic lactone with structural similarity to spinosad, have shown that ivermectin makes direct associations (by hydrogen bonding and van der Waals interactions) with TM1, TM2 and TM3. ${ }^{26}$ This finding is consistent with spinosad acting via an allosteric transmembrane site distinct from the conventional agonist binding site, ${ }^{24}$ and it is thus likely that the F238del mutation confers resistance by directly modifying the spinosad binding site.

It is notable that neither the G275E mutation or exon 3 skipping, previously identified in strains of T. absouta from Brazil and Portugal, respectively, were observed in UK strains of T. absoluta, suggesting that the mutations identified in our study arose de novo in UK populations rather than being imported via transfer of insects on plant material or packaging from outside the UK. The application of spinosad via the irrigation system in the UK, together with the redundancy of the nicotinic acetylcholine receptor (nAChR) $\alpha 6$ subunit, in which any non-functionalizing mutation will confer resistance, likely facilitated the rapid evolution of resistance.

Two of the strains tested in our study also showed tolerance to chlorantraniliprole, while the levels of tolerance displayed were relatively modest selection of one of these strains rapidly resulted in a line with potent resistance to this compound. This finding suggests that resistance alleles were almost certainly present in the population prior to selection. Sequencing of a region of the RyR encompassing the sites of known resistance mutations confirmed this and revealed that a mutation leading to a G4903V substitution present in the parental strain at low frequency had reached fixation in the selected line. This mutation has recently been reported in $T$. absoluta strains from Greece and Italy ${ }^{8}$, suggesting that T. absoluta with this resistance allele may have been imported into the UK. The functional significance of the G4903V substitution has been confirmed in vitro using radioligand binding studies and in vivo by CRISPR/CAS9 genome editing in Drosophila. ${ }^{13}$ In the case of the latter genome modified flies bearing the mutation exhibited high levels of resistance to both the diamides flubendiamide (91-fold) and chlorantraniliprole (195-fold) when compared to flies of the same genetic background without the mutation. ${ }^{13}$

Our results have significant applied implications in relation to the control of T. absoluta in the UK. Spinosad performs a key role in the IPM strategy introduced against this pest in 2013, and is applied at the start of the season to provide control while the natural enemy M. pygmaeus is becoming established. ${ }^{3}$ If necessary, a high-volume spray of chlorantraniliprole is applied as a second line of defence later in the season to keep the pest and predator populations in balance. ${ }^{3}$ The fact that we identified one strain with resistance mechanisms to both spinosad and chlorantraniliprole is concerning as it demonstrates that T. absoluta with resistance to both compounds can readily evolve and are viable. It would be useful to screen UK populations of T. absoluta more widely for the resistance mechanisms identified in this study. If such monitoring indicates that, as suggested by our study, spinosad resistance is widespread, then we suggest that use of this insecticide is suspended for a period to examine if susceptibility is restored to populations in the absence of selection. The likelihood of this is dependent on any fitness costs associated with the resistance mechanisms we have identified. In D. melanogaster no evidence of fitness costs were identified under laboratory conditions in a $\mathrm{D} \alpha 6$ null mutant, suggesting the costs of loss of function mutations in this subunit may be minimal. ${ }^{16}$ However, work on a spinosad-selected strain of T. absoluta found that resistance was unstable in the absence of spinosad selection and dropped rapidly over relatively few (less than ten) generations. ${ }^{27}$ In our study we observed high levels of spinosad resistance in a strain (EVH16) collected from a nursery where this compound had not been used for a year, suggesting that the exon 4 skipping identified in this strain may have a low fitness penalty. However, additional work is required to clarify fitness costs associated with this mutation and the others identified in this study in both the $\alpha 6 \mathrm{nAChR}$ subunit and the RyR under field-realistic conditions.

Mutations conferring resistance to chlorantraniliprole were only identified in a single strain in our study, suggesting that with effective resistance management it may be possible to prevent the further development of resistance to this compound. This would require the rotation of this insecticide with insecticides of alternative modes of action and/or the use of alternative control methods. In regards to the latter recent trials in the UK using a synthetic sex pheromone as a mating disruption strategy against $T$. absoluta have provided encouraging results ${ }^{28}$ and may allow the frequency of insecticide applications to be significantly reduced. However, our study clearly illustrates the danger of complacency and we suggest that the control of T. absoluta in the UK (and worldwide) should continue to rely on the use of a range of integrated approaches in order to reduce selection pressure on any single control to prevent or slow the emergence of resistance.

\section{ACKNOWLEDGEMENTS}

This work was funded by $\mathrm{PhD}$ studentship awards from the Agriculture and Horticulture Development Board (AHDB) (CP 162) and the Biotechnology and Biological Sciences Research Council (BBSRC) (grant number: 1096240). ER was supported by funding from the European Union's Seventh Framework Programme for research, technological development and demonstration under the STomP project, grant agreement no. 219262 (ARIMNet).

\section{SUPPORTING INFORMATION}

Supporting information may be found in the online version of this article.

\section{REFERENCES}

1 Desneux N, Luna M, Guillemaud T and Urbaneja A, The invasive South American tomato pinworm, Tuta absoluta, continues to spread in Afro-Eurasia and beyond: the new threat to tomato world production. J Pest Sci 84:403-408 (2011).

2 Cuthbertson AGS, Mathers JJ, Blackburn LF, Korycinska A, Luo W, Jacobson RJ etal., Population development of Tuta absoluta (Meyrick) (Lepidoptera: Gelechiidae) under simulated UK glasshouse conditions. Insects 4:185-197 (2013).

3 Jacobson RJ and Howlett $\mathrm{P}, \mathrm{A}$ robust IPM programme for Tuta absoluta. Horticultural Development Company; Factsheet 02/14. [Online]. Available: https://horticulture.ahdb.org.uk/publication/0214robust-ipm-programme-tuta-absoluta (2014).

4 Campos MR, Silva TBM, Silva WM, Silva JE and Siqueira HAA, Spinosyn resistance in the tomato borer Tuta absoluta (Meyrick) (Lepidoptera: Gelechiidae). J Pest Sci 88:405-412 (2015).

5 Silva WM, Berger M, Bass C, Williamson M, Moura DMN, Ribeiro LMS et al., Mutation (G275E) of the nicotinic acetylcholine receptor $\alpha 6$ subunit is associated with high levels of resistance to spinosyns in Tuta absoluta (Meyrick) (Lepidoptera: Gelechiidae). Pest Biochem Physiol 131:1-8 (2016). 
6 Zimmer CT, Garrood WT, Puinean AM, Eckel-Zimmer M, Williamson MS, Davies TG et al., A CRISPR/Cas9 mediated point mutation in the alpha 6 subunit of the nicotinic acetylcholine receptor confers resistance to spinosad in Drosophila melanogaster. Insect Biochem Mol Biol 73:62-69 (2016).

7 Berger M, Puinean AM, Randall E, Zimmer CT, Silva WM, Bielza P, et al., Insecticide resistance mediated by an exon skipping event. $\mathrm{Mol}$ Ecol 25: $5692-5704$ (2016).

8 Roditakis E, Steinbach D, Moritz G, Vasakis E, Stavrakaki M, Ilias A, et al., Ryanodine receptor point mutations confer diamide insecticide resistance in tomato leafminer, Tuta absoluta (Lepidoptera: Gelechiidae). Insect Biochem Mol Biol 80:11-20 (2017).

9 Roditakis E, Vasakis E, Grispou M, Stavrakaki M, Nauen R, Gravouil M et al., First report of Tuta absoluta resistance to diamide insecticides. J Pest Sci 88:9-16 (2015).

10 Silva JE, Ribeiro LMS, Vinasco N, Raul NCG and Siqueira HA, Field-evolved resistance to chlorantraniliprole in the tomato pinworm Tuta absoluta: inheritance, cross-resistance profile, and metabolism. J Pest Sci:1 - 11 (2019). https://doi.org/10.1007/s10340018-1064-z.

11 Guo L, Liang P, Zhou X and Gao X, Novel mutations and mutation combinations of ryanodine receptor in a chlorantraniliprole resistant population of Plutella xylostella (L.). Sci Rep 4:6924 (2014).

12 Troczka B, Zimmer CT, Elias J, Schorn C, Bass C, Davies TGE, et al., Resistance to diamide insecticides in diamondback moth, Plutella xylostella (Lepidoptera: Plutellidae) is associated with a mutation in the membrane-spanning domain of the ryanodine receptor. Insect Biochem Mol Biol 42(11): $873-880$ (2012).

13 Douris V, Papapostolou KM, Ilias A, Roditakis E, Kounadi S, Riga M, et al., Investigation of the contribution of RyR target-site mutations in diamide resistance by CRISPR/Cas9 genome modification in Drosophila. Insect Biochem Mol Biol 87:127-135 (2017).

14 Zuo YY, Wang H, Xu YJ, Huang JL, Wu SW, Wu YD et al., CRISPR/Cas9 mediated G4946E substitution in the ryanodine receptor of Spodoptera exigua confers high levels of resistance to diamide insecticides. Insect Biochem Mol Biol 89:79-85 (2017).

15 Roditakis E, Skarmoutsou C, Staurakaki M, Martínez-Aguirre MR, García-Vidal L, Bielza P, et al., Determination of baseline susceptibility of European populations of Tuta absoluta (Meyrick) to indoxacarb and chlorantraniliprole using a novel dip bioassay method. Pest Manag Sci 69:217-227 (2013).

16 Perry T, McKenzie JA, Batterham P, Perry T, JA MK and Batterham P, A knockout strain of Drosophila melanogaster confers a high level of resistance to spinosad. Insect Biochem Mol Biol 37:184-188 (2007).

17 Baxter SW, Chen M, Dawson A, Zhao JZ, Vogel H, Shelton AM, et al., Mis-spliced transcripts of nicotinic acetylcholine receptor alpha6 are associated with field evolved spinosad resistance in Plutella xylostella (L.). PLoS Genet 6:e1000802 (2010).

18 Rinkevich FD, Chen M, Shelton AM and Scott JG, Transcripts of the nicotinic acetylcholine receptor subunit gene Pxyl $\alpha 6$ with premature stop codons are associated with spinosad resistance in diamondback moth, Plutella xylostella. Invert Neurosci 10:25-33 (2010).

19 Hsu JC, Feng HT, Wu WJ, Geib SM, Mao CH and Vontas J, Truncated transcripts of nicotinic acetylcholine subunit gene $B d \alpha 6$ are associated with spinosad resistance in Bactrocera dorsalis. Insect Biochem $\mathrm{Mol}$ Biol 42:806-815 (2012).

20 Wan Y, Yuan G, He B, Xu B, Xie W, Wang S et al., Focc $\alpha 6$, a truncated $\mathrm{nAChR}$ subunit, positively correlates with spinosad resistance in the western flower thrips, Frankliniella occidentalis (Pergande). Insect Biochem Mol Biol 99:1 - 10 (2018).

21 Wang $\mathrm{H}-\mathrm{T}$, Tsai C-L and Chen M-E, Nicotinic acetylcholine receptor subunit $\alpha 6$ associated with spinosad resistance in Rhyzopertha dominica (Coleoptera: Bostrichidae). Pest Biochem Physiol 148:68-73 (2018).

22 Wang J, Wang X, Lansdell SJ, Zhang J, Millar NS and Wu Y, A three amino acid deletion in the transmembrane domain of the nicotinic acetylcholine receptor $\alpha 6$ subunit confers high-level resistance to spinosad in Plutella xylostella. Insect Biochem Mol Biol 71:29-36 (2016).

23 Bao WX, Narai Y, Nakano A, Kaneda T, Murai T and Sonoda S, Spinosad resistance of melon thrips, Thrips palmi, is conferred by G275E mutation in $\alpha 6$ subunit of nicotinic acetylcholine receptor and cytochrome P450 detoxification. Pest Biochem Physiol 112:51-55 (2014).

24 Puinean AM, Lansdell SJ, Collins T, Bielza P and Millar NS, A nicotinic acetylcholine receptor transmembrane point mutation (G275E) associated with resistance to spinosad in Frankliniella occidentalis. J Neurochem 124:590-601 (2013).

25 Hiruta E, Aizawa M, Nakano M and Sonoda S, Nicotinic acetylcholine receptor $\alpha 6$ subunit mutation (G275V) found in a spinosad-resistant strain of the flower thrips, Frankliniella intonsa (Thysanoptera: Thripidae). J Pest Sci 43:272-276 (2018).

26 Hibbs RE and Gouaux E, Principles of activation and permeation in an anion-selective Cys-loop receptor. Nature 474:54-60 (2011).

27 Campos MR, Rodrigues ARS, Silva WM, Silva TBM, Silva VRF, Guedes RNC et al., Spinosad and the tomato borer Tuta absoluta: a bioinsecticide, an invasive pest threat, and high insecticide resistance. PLoS One 9:e103235 (2014).

28 AHDB, New Mating Disruption Technique Offers Hope for Tuta Absoluta Control. [Online] (2017). Available: https://horticultureahdborguk/ news-item/new-mating-disruption-technique-offers-hope-tutaabsoluta-control [11 December 2018]. 